\title{
Mutual Capacitive Calculation for Touch Panel Design
}

\author{
Jiumming $\operatorname{Lin}^{1, a^{*}}$, Mungwei $\operatorname{Lin}^{2, b}$ and Chenghung $\operatorname{Lin}^{3, c}$ \\ ${ }^{1}$ Department of Electronic Engineering, Chung-Hua University, Taiwan \\ ${ }^{2} \mathrm{Ph}$. D. Program in Engineering Science, Chung-Hua University, Taiwan

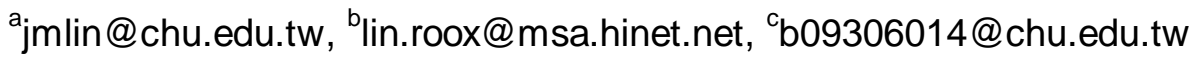

Keywords: Touch panel; Self capacitance; Mutual capacitance; Maxwell capacitance matrix

\begin{abstract}
This paper took four kinds of circuit layouts for mutual capacitive touch panel design, such as diamond, metal mesh, strip, and strip-style semi-ladder tree. One could see the electrodes overlapping area for the patterns of mesh and strip is the largest, but its percentage change of mutual capacitance is the smallest (-25.4\%). On the other hand, the electrodes overlapping area of sensing electrodes with hexagonal lace pattern and driving electrodes with mesh pattern is the smallest. But its percentage change is the largest $(-47.6 \%)$, and so it is easy for discrimination, so do the IC driver design is easy.
\end{abstract}

\section{Introduction}

The touch panel can simplify the human-machine interface by using finger to touch the screen to communicative the information on the internet. It can also make the device be lighter, flatter, shorter and smaller. The operation principles of touch panel can be divided into two kinds, such as resistive and capacitive types [1-5]. The capacitive electrodes have many shapes, such as mesh, strip, diamond, and strip-style semi-ladder tree. In this paper the self and mutual capacitances for each pattern of sensing and driving electrodes can be analyzed using COMSOL 3D simulation software to check which one is the best for the design [6-7]. One could see the electrodes overlapping area for the patterns of mesh and strip is the largest, but its percentage change of mutual capacitance is the smallest $(-25.4 \%)$. On the other hand, the electrodes overlapping area of sensing electrodes with hexagonal lace pattern and driving electrodes with mesh pattern is the smallest. But its percentage change is the largest $(-47.6 \%)$, and so it is easy for discrimination, so do the IC driver design is easy [7-11].

The other contains of this paper are as follows: the second section is for the operation principle of mutual capacitance sensing technology. Section 3 briefs the mutual capacitance simulation. The final section is conclusion.

\section{Operation Principle of Capacitive Touch Panel}

Structure of Capacitive Touch Panel. The capacitive touch panel applies a transparent conductive layer of ITO (Antimony Tin Oxide) with conductivity as 1200-2000 $\Omega / \square$ (ohm/sqr) on the upper surface of glass as shown in Fig. 1 [11]. In Fig. 2 four ITO electrodes are coated at the corners of glass panel, thus the electric fields can be set on the surface of the panel [2]. An IC chip drives a sinusoidal or a pulsed signal (with frequency from $25 \mathrm{~Hz}$ to $200 \mathrm{KHz}$ ) to those four electrodes to measure the values of the surface capacitances. When a finger (grounding) touches the panel, then the finger and the electrodes at corners on the glass panel can result in four parasitic capacitances as shown in Fig. 3. Those parasitic capacitances can be arranged in a pair of Wheatstone bridge circuits, and the driver IC can determine those parasitic capacitances and the touch point coordinate of finger in either $\mathrm{x}$ - or $\mathrm{y}$-axis. This kind of touch panel is only available for a single touch point determination. The projective sensing method has two types by using either self or mutual capacitance. The self capacitance sensing method is to measure the capacitance between ground and sensing (or driving) electrode as shown in Fig. 3a. While the mutual capacitance sensing method is to measure the capacitance between the sensing and driving electrode as shown in Fig. 3b. This method can eliminate the ghost problem caused by using 
self capacitance sensing method for multiple touching points determination, and was studied in this paper.

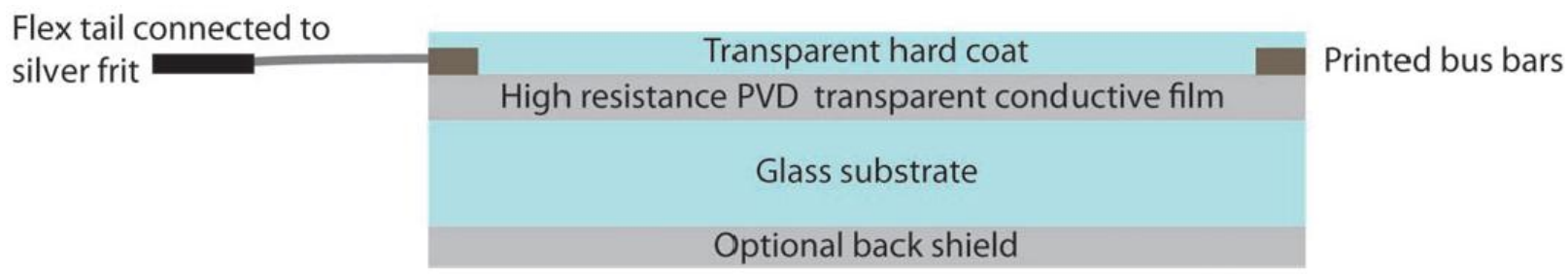

Figure 1. Capacitive touch panel structure

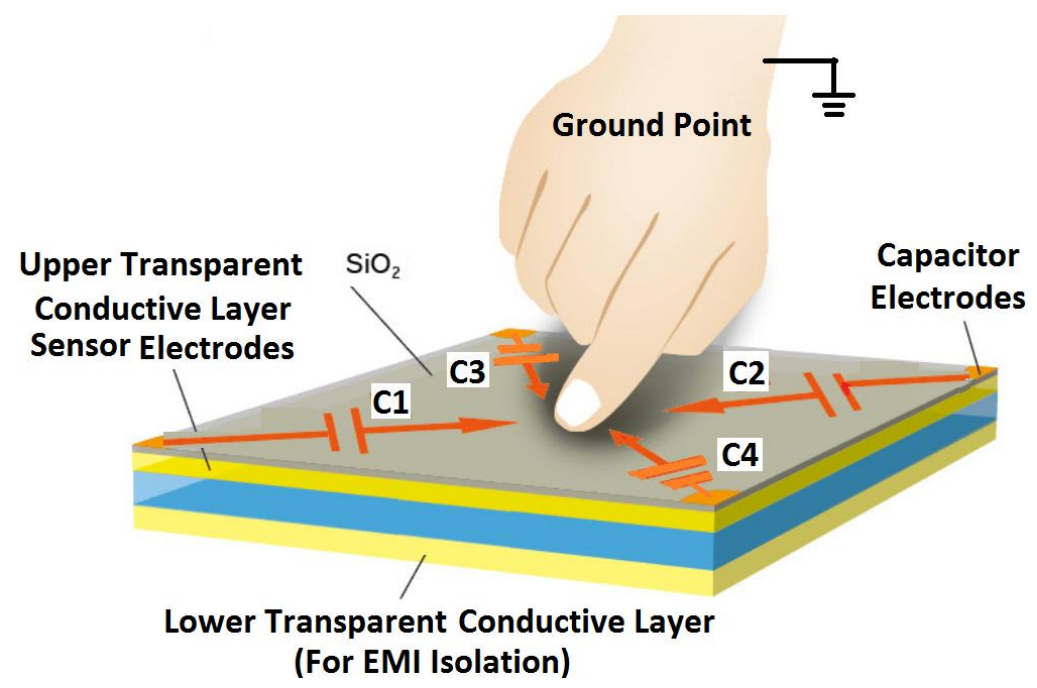

Figure 2. Capacitances with finger and corner electrodes
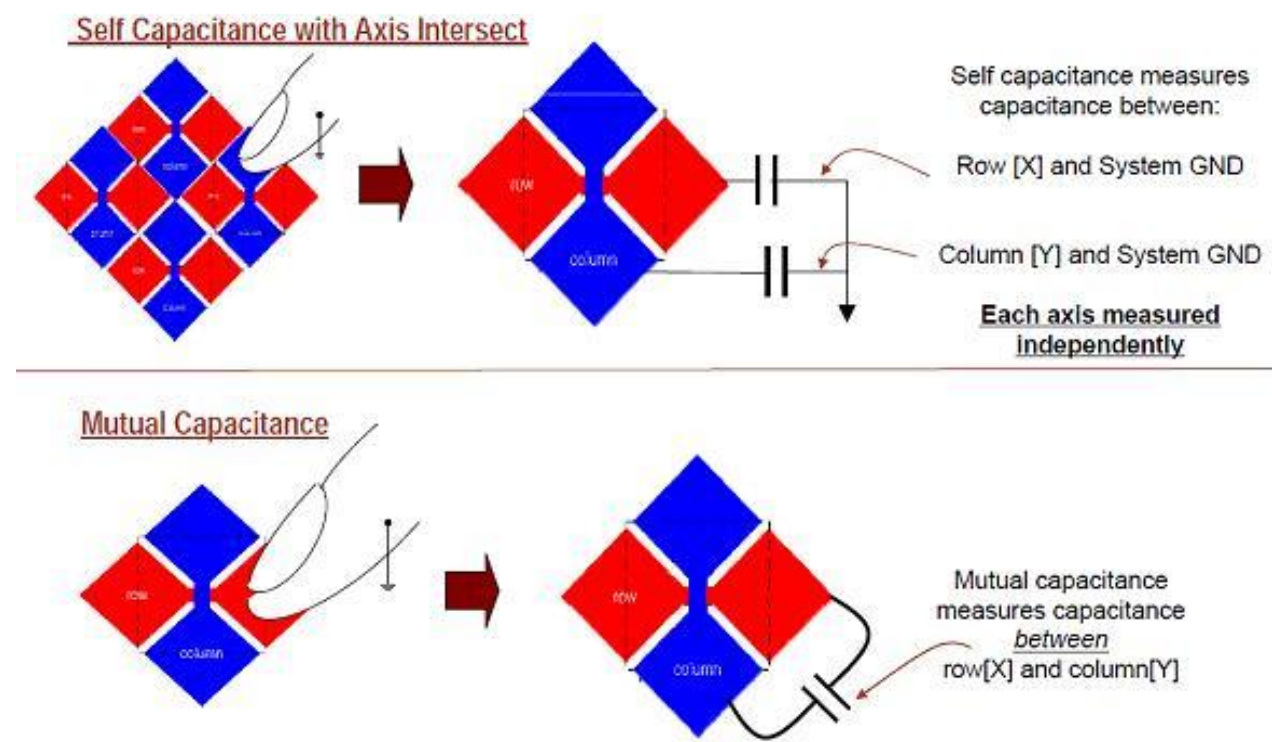

Figure 3. Types of capacitance sensing methods (a) Self capacitance (b) Mutual capacitance

\section{Mutual Capacitance Analysis Method}

This paper took four kinds of layout patterns for mutual capacitance analysis, such as diamond, strip-style semi-ladder tree, metal mesh, and strip as respectively shown in Figs. 4a-4d. The overlapping areas of the electrodes of SP1-SP4 are respectively as $0.16 \mathrm{~mm}^{2}, 6.5 \mathrm{~mm}^{2}, 0.056 \mathrm{~mm}^{2}$ and $6.5 \mathrm{~mm}^{2}$. The Maxwell capacitance matrix is applied to find the self and mutual capacitances [4]. An 
example of two conductors respectively in $\mathrm{X}$-axis and $\mathrm{Y}$-axis are applied for illustration as follows: If $\mathrm{V}$ and $\mathrm{Q}$ are the voltage and charge of the conductors, and the self and mutual capacitances are as shown in Fig. 5. Let the system is in an isolated environment, then the total charge of the system is 0 , and the relationships of charges and capacitances can be equated as follows.
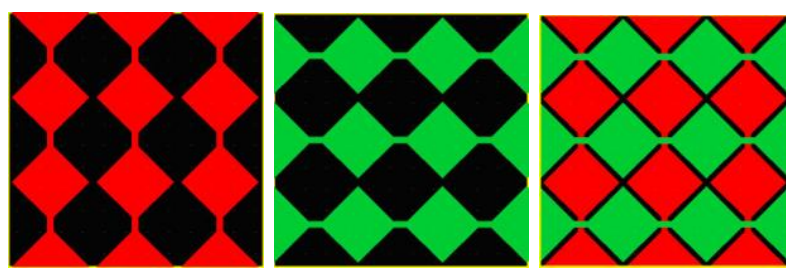

SP1 patterns (both sensing (red) and driving (green) electrodes are with diamond patterns)
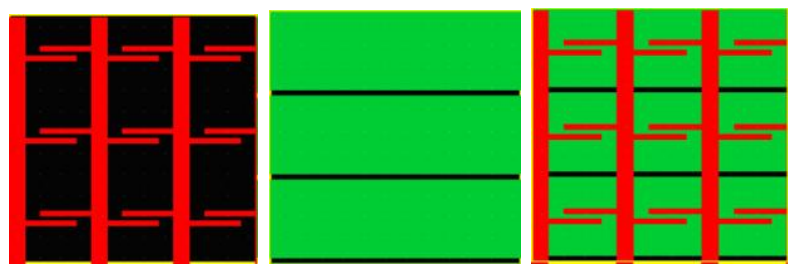

SP2 patterns (the sensing (red) electrodes are with strip-style semi-ladder tree pattern, and the driving (green) electrodes are with strip pattern)

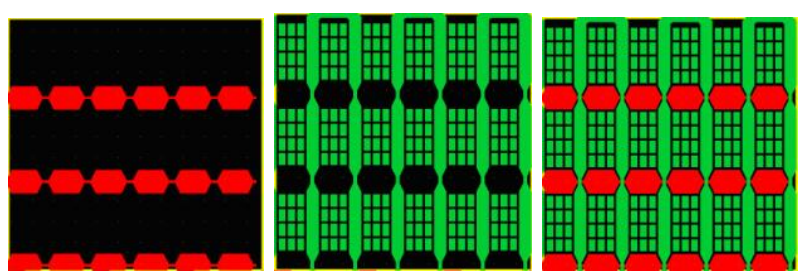

SP3 patterns (sensing (red) electrodes are with hexagonal lace pattern and driving (green) electrodes with mesh pattern)
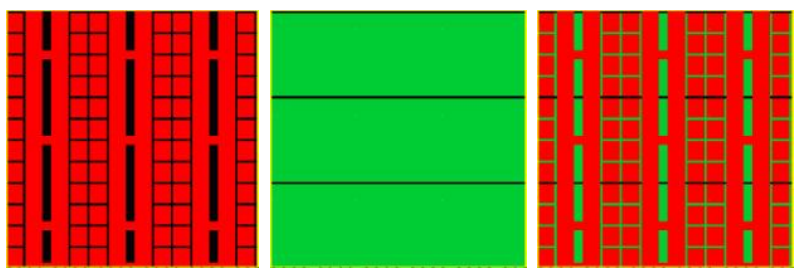

d. SP4 patterns (the sensing (red) and driving (green) electrodes are respectively with mesh and strip patterns)

Figure 4. Sensing (red) and driving (green) electrodes of SP1-SP4 patterns

$$
\begin{aligned}
& \mathrm{Q} 1=\mathrm{C} 11 \mathrm{~V} 1+\mathrm{C} 12(\mathrm{~V} 1-\mathrm{V} 2)+\mathrm{C} 13(\mathrm{~V} 1-\mathrm{V} 3)+\mathrm{C} 14(\mathrm{~V} 1-\mathrm{V} 4) \\
& \mathrm{Q} 2=\mathrm{C} 22 \mathrm{~V} 2+\mathrm{C} 21(\mathrm{~V} 2-\mathrm{V} 1)+\mathrm{C} 23(\mathrm{~V} 2-\mathrm{V} 3)+\mathrm{C} 24(\mathrm{~V} 2-\mathrm{V} 4) \\
& \mathrm{Q} 3=\mathrm{C} 33 \mathrm{~V} 3+\mathrm{C} 31(\mathrm{~V} 3-\mathrm{V} 1)+\mathrm{C} 32(\mathrm{~V} 3-\mathrm{V} 2)+\mathrm{C} 34(\mathrm{~V} 3-\mathrm{V} 4) \\
& \mathrm{Q} 4=\mathrm{C} 44 \mathrm{~V} 4+\mathrm{C} 41(\mathrm{~V} 4-\mathrm{V} 1)+\mathrm{C} 42(\mathrm{~V} 4-\mathrm{V} 2)+\mathrm{C} 43(\mathrm{~V} 4-\mathrm{V} 3)
\end{aligned}
$$

Re-grouping the same voltages in each equation one has the following results:

$$
\begin{aligned}
& \mathrm{Q} 1=(\mathrm{C} 11+\mathrm{C} 12+\mathrm{C} 13+\mathrm{C} 14) \mathrm{V} 1-\mathrm{C} 12 \mathrm{~V} 2-\mathrm{C} 13 \mathrm{~V} 3-\mathrm{C} 14 \mathrm{~V} 4 \\
& \mathrm{Q} 2=-\mathrm{C} 21 \mathrm{~V} 1+(\mathrm{C} 21+\mathrm{C} 22+\mathrm{C} 23+\mathrm{C} 24) \mathrm{V} 2-\mathrm{C} 23 \mathrm{~V} 3-\mathrm{C} 24 \mathrm{~V} 4 \\
& \mathrm{Q} 3=-\mathrm{C} 31 \mathrm{~V} 1-\mathrm{C} 32 \mathrm{~V} 2+(\mathrm{C} 31+\mathrm{C} 32+\mathrm{C} 33+\mathrm{C} 34) \mathrm{V} 3-\mathrm{C} 34 \mathrm{~V} 4 \\
& \mathrm{Q} 4=-\mathrm{C} 41 \mathrm{~V} 1-\mathrm{C} 42 \mathrm{~V} 2-\mathrm{C} 43 \mathrm{~V} 3+(\mathrm{C} 41+\mathrm{C} 42+\mathrm{C} 43+\mathrm{C} 44) \mathrm{V} 4
\end{aligned}
$$


An example of SP4 pattern as shown in Fig. 6 was applied for illustration. The self and mutual capacitances of simulations are listed in Table 1 for comparison. Thus $\mathrm{C}_{11}$ is $1.89512 \mathrm{pF}$, and $\mathrm{C}_{12}$ is $0.02563 \mathrm{pF}$, so $\mathrm{C}_{12}$ is much smaller than $\mathrm{C}_{11}$.

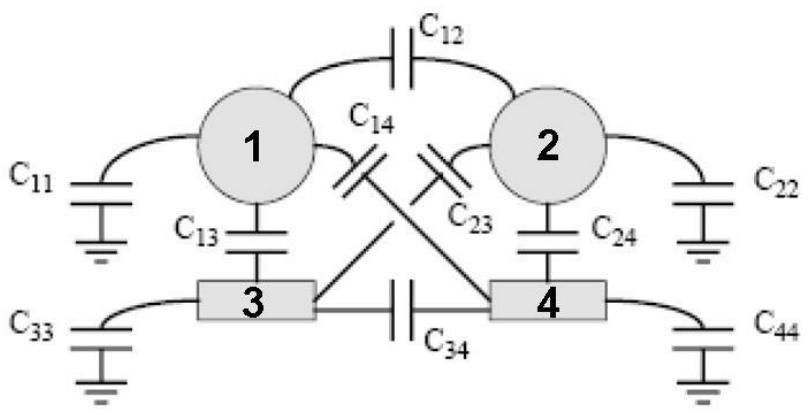

Figure 5. Self and mutual capacitances

\section{Simulation Results of Mutual Capacitance}

The mutual capacitances of the four kinds of electrode patterns would be checked at the node for the touch panel design. The simulation results of the mutual capacitances using COMSOL 3D simulation software [5-6], i.e. the average values without and without grounding copper cylinder (used as finger), as well as their changes in magnitudes and percentages, are listed in Table 2.

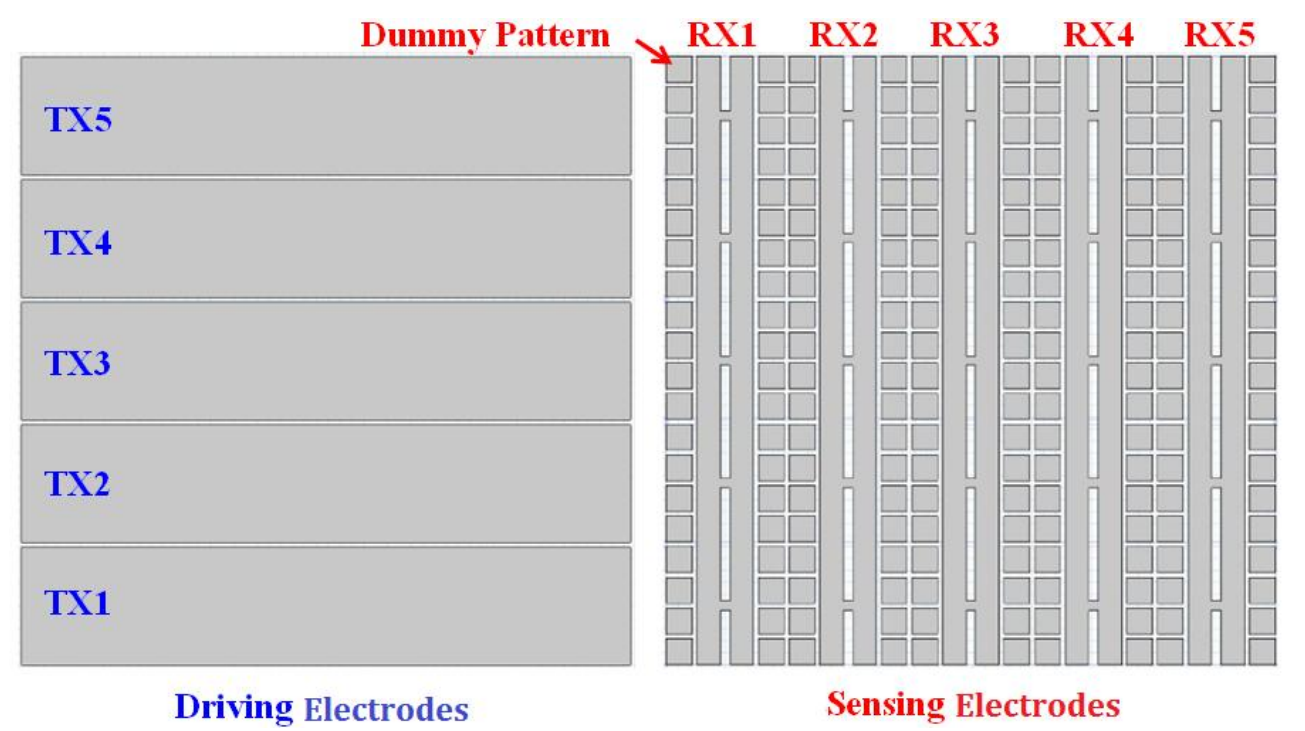

Figure 6. Patterns of sensing and driving electrodes of SP4

Table 1 The self and mutual capacitances for SP4 pattern

\begin{tabular}{|c|c|c|c|c|}
\hline Capacitances (pF) & Column 1 & Column 2 & Column 3 & Column 4 \\
\hline Row 1 & 1.89512 & 0.02563 & 0.93474 & 0.93475 \\
\hline Row 2 & 0.02563 & 1.89463 & 0.93454 & 0.93446 \\
\hline Row 3 & 0.93474 & 0.93454 & 2.36463 & 0.49535 \\
\hline Row 4 & 0.93475 & 0.93446 & 0.49535 & 2.36457 \\
\hline
\end{tabular}

Thus, one can see the electrodes overlapping area of SP4 is the largest, but its percentage change of mutual capacitances is the smallest (-25.4\%), thus the IC driver design is very hard. On the other hand, the electrodes overlapping area of SP3 is the smallest, but its percentage change of the mutual capacitances is the largest (-47.6\%), and so it is easy for discrimination, so do the IC driver design is easy [7-11]. 
Table 2 Simulation results of the mutual capacitances of the four patterns

\begin{tabular}{|c|c|c|c|c|}
\hline Items of Mutual Capacitances & SP1 & SP2 & SP3 & SP4 \\
\hline Average values without copper cylinder $(\mathbf{p F})$ & 0.587 & 0.894 & 0.442 & 1.372 \\
\hline Average values with copper cylinder $\mathbf{( p F )}$ & 0.375 & 0.617 & 0.233 & 1.024 \\
\hline Changes of mutual capacitance $(\mathbf{p F})$ & 0.212 & 0.278 & 0.209 & 0.349 \\
\hline Percentage changes of mutual capacitances $\mathbf{( \% )}$ & -36.1 & -31.1 & -47.6 & -25.4 \\
\hline
\end{tabular}

\section{Summary}

This paper took four kinds of circuit layouts for mutual capacitive touch panel design, such as metal mesh, strip, diamond, and strip-style semi-ladder tree. In this paper the self and mutual capacitances for each pattern of sensing and driving electrodes can be analyzed using COMSOL 3D simulation software to check which one is the best for the design. One could see the electrodes overlapping area for the patterns of mesh and strip is the largest, but its percentage change of mutual capacitance is the smallest (-25.4\%). On the other hand, the electrodes overlapping area of sensing electrodes with hexagonal lace pattern and driving electrodes with mesh pattern is the smallest. But its percentage change is the largest (-47.6\%), and so it is easy for discrimination, so do the IC driver design is easy.

\section{Acknowledgements}

National Science Council supported this research with the grants: MOST 104-2221-E-216-021.

\section{References}

[1] J. Fraden, Handbook of Modern Sensors: Physics, Designs, and Applications, third ed., Springer-Verlag, New York, 2003.

[2] Information on http://www.ntss-tech.com.tw/touch-skd-panel/optical-touch-panel/

[3] P. Kearney, The PDA Challenge - Met by the AD7873 Resistive-Touch-Screen Controller ADC, Information on http://www.analog.com/library/analogdialogue/archives/35-04/touchscreen/

[4] E.D. Lorenzo, the Maxwell Capacitance Matrix, White Paper, Fast Field Solvers, Vimercate (MB) Italy, 2011.

[5] A. Qaiser, Designing a Capacitive Sensor Using COMSOL, Application Note, Michigan State University, 2010.

[6] L.K. Baxter, Capacitive Sensors: Design and Applications, Wiley-Blackwell, New Jersey, 1996. COMSOL Multiphysics, User's Guide Version 4.1, 2010.

[7] Information on http://www.touchscreenmagazine.nl/

[8] Avago Technologies, Touch Controller AMRI-5100 (PTHD) Introduction, 2014.

[9] FocalTech Systems Co., Ltd., FT5x06 datasheet, True Multi-Touch Capacitive Touch Panel Controller, Version 0.2, June 21, 2010.

[10] A.G. Podbelski, Cypress Semiconductor, Cypress' CapSense Sigma-Delta Algorithm, 2007.

[11]C. Bauman, How to select a surface-capacitive touch-screen controller, SID. 12 (2007) 32-36. 